Review article

Paediatrics Today 2016;12(1):3-15

DOI $10.5457 / \mathrm{p} 2005-114.131$

\title{
A PEEK INTO THE UTERUS - MRI OF THE FETAL CNS
}

\author{
Tina VIPOTNIK VESNAVER* \\ Clinical Institute of Radiology \\ University Clinical Centre Ljubljana
}

*Corresponding author:
tinavipotnik@yahoo.com
Tel.: + 38615228530
Fax.: + 38615222482

Received: November 10, 2014

Accepted: December 14, 2014

Key words: Prenatal diagnosis • MR imaging of the fetal CNS - Normal development - Developmental abnormalities.

\begin{abstract}
The purpose of this article is to provide an overview of MR imaging of the fetal central nervous system, and to briefly discuss recent advances. Fetal MRI has been commonly used throughout the world for over 20 years. Compared to US it has better contrast resolution, allows direct visualization of both hemispheres and the additional limitations of sonography do not affect the quality of MRI. There are no known adverse short-term or long-term side effects of MRI in children, who were imaged in the fetal period. The standard protocol for fetal MRI consists of single-shot rapid acquisition T2 weighted sequences in three planes, fast spoiled gradient recalled $\mathrm{T} 1$ weighted sequence, $\mathrm{T} 2$ gradient echo sequence and diffusion weighted sequence in the axial plane. The clinical applications of advanced MRI techniques are still limited. Fetal MRI is performed to confirm abnormalities detected on US, and to exclude additional pathology. Interpreting fetal MRI images requires understanding of normal fetal CNS development and one should also evaluate other fetal structures, the umbilical cord and placenta. The most common indication for MR imaging of the fetal CNS is mild ventriculomegaly. Other common indications, that are also discussed in the article, include commissural abnormalities, infratentorial abnormalities, cortical malformations, spinal pathology and complications of monochorionic twin pregnancy. Conclusion Fetal MRI provides important information in prenatal counseling, therefore familiarity with the applications and limitations of fetal MRI, and multidisciplinary collaboration remain essential for the radiologist involved in prenatal imaging.
\end{abstract}

\section{Introduction}

Fetal magnetic resonance imaging (MRI) is a diagnostic imaging modality that has been commonly used in the world for over 20 years. The first case of MRI performed during pregnancy was described in 1983 (1), but initially it was done primarily in the setting of maternal and placental pathology (2). On standard echo-planar sequences, images of the fetus were degraded due to motion artifacts, therefore the imaging was only used for volumetric analyses (3). In the early nineties, single-shot rapid acquisition sequence with refocused echoes was developed, which enabled a profound insight into the uterus (4). Singleshot rapid acquisition sequence with refocused echoes is a high-quality T2-weighted sequence that has a slice acquisition time of less than a second, effectively "freezing" fetal motion (5).

In this review we discuss the safety and technical aspects, utility and limitations of fetal MR imaging, the normal appearance of the fetal brain in different stages of its development, and focus on the most common patho- 
logical findings. We will briefly address the latest developments in the field of fetal MRI.

\section{Fetal safety}

Several studies have been performed and so far have failed to show any adverse short- or long-term side effects of $1.5 \mathrm{~T}$ MR imaging on fetal and postnatal development (6-9). Although most fetal MR studies are performed on a $1.5 \mathrm{~T}$ scanner, fetal MRI is also performed on $3 \mathrm{~T}$ scanners, as they provide better anatomical detail. In the guidelines prepared in 2014 by Patenaude et al. on the use of MRI in obstetric patients it is stated that "Fetal magnetic resonance imaging is safe at 3.0 tesla or less, during the second and third trimesters"(8). Due to the theoretical risk during organogenesis, when the fetus is also very small, it is advisable to avoid MRI during the first trimester. Some animal studies have shown the possibility of teratogenic effects in early prenatal development, but the applicability of these animal models to humans has been questioned (8-11). Imaging younger fetuses can also be a challenge due to the small dimensions and fetal movements $(12,13)$. In clinical practice, fetal MRI is therefore usually performed after 18 weeks of pregnancy. Paramagnetic contrast agent gadolinium (Gd) administration to a pregnant women is not recommended unless the potential benefit outweighs the unknown, but potentially real risk of the long-term exposure of the developing fetus to free $\mathrm{Gd}$ ions (7). The potential risk of acoustic damage due to noise generated by the MR scanner is negligible. Prudence is recommended when a MR study is indicated (also for maternal pathology) in the first trimester (14).

\section{Fetal MR techniques}

In our institution we perform fetal MRI on a Siemens Aera 1.5T MR scanner with the use of an 18 channel phased array body coil to in- crease coverage of the fetal region of interest, and to increase signal to noise ratio (SNR). We ask the mother to fast $4 \mathrm{~h}$ prior to examination to reduce bowel peristalsis and fetal motion $(12,13)$, but this practice differs between institutions. Before the patient enters the scanner, we advise her to empty her bladder. During the study, which lasts around $30 \mathrm{~min}$, the mother lies supine. If she feels uncomfortable or the uterus compresses the inferior vena cava, she can lie in the left decubitus position, but that may degrade the image quality.

To assess the fetal position, we start the examination with a 3D localizer, using singleshot rapid acquisition T2 weighted sequence, with 6-8 mm slice thickness, 1-2 mm gap and a large field of view. We continue the examination with single-shot rapid acquisition T2 weighted sequences in the axial, coronal and sagittal planes. With this sequence, one image can be obtained in less than a second, thus minimizing motion artifacts, which is particularly important during earlier pregnancy when the fetus is small, and in cases when the fetal head is not yet engaged in the pelvis. The standard slice thickness when imaging brain is $3-4 \mathrm{~mm}$, with no gap. In spine imaging, $2 \mathrm{~mm}$ slice thickness is required due to the smaller structures. T2 weighted sequences have good contrast and spatial resolution, and are used as standard sequences for assessment of fetal anatomy and pathology.

A fast spoiled gradient recalled T1 weighted sequence has lower SNR and requires a longer acquisition time (approximately 18 seconds) with the consequent higher susceptibility to fetal and maternal motion (12). Slice thickness is $5 \mathrm{~mm}$ with a $1 \mathrm{~mm}$ gap. T1 weighted images in the axial plane are acquired during maternal breath hold. They are used primarily for detection of blood, calcification and fat. Gradient echo-planar T2 weighted images help us detect hemorrhage. Images in the axial plane are acquired during a single maternal breath hold. Structural imaging is the mainstay of fetal MR imag- 

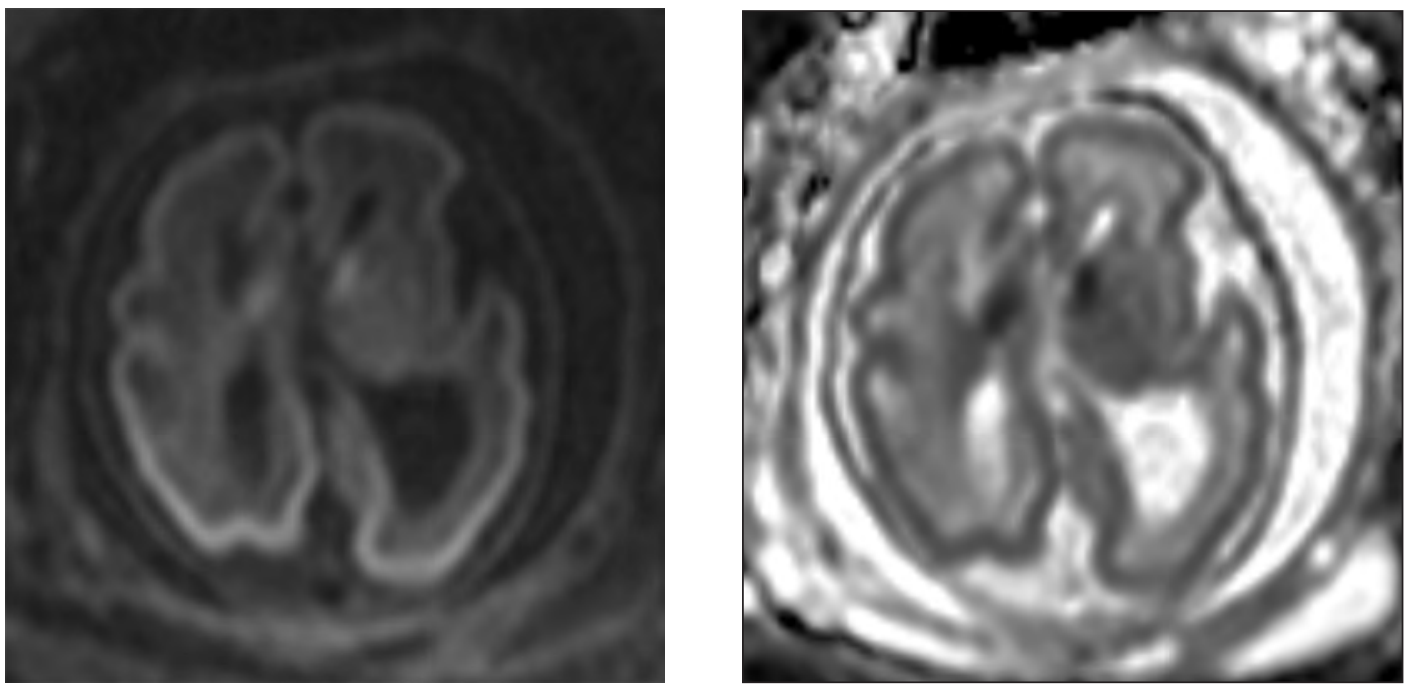

Fig. $1 D W I$ sequence (left) and ADC map (right), with lower diffusion and the corresponding lower apparent diffusion coefficient $(A D C)$ values in the caudothalamic groove and cerebral cortex - a normal finding. A case of a fetus with isolated ventriculomegaly.

ing, but other modalities may also be a valuable tool in the diagnostic process. Diffusion weighted imaging is being used more and more commonly in standard fetal protocols. It is primarily indicated for detection of acute hypoxic ischemic lesions in fetuses (15) (Fig. 1). With diffusion tensor imaging and MR tractography, visualization of the main neuronal pathways is possible, thus enabling new insights into normal and pathological brain development (16). Single voxel spectroscopy is a non-invasive method for assessment of brain metabolism (17). Its clinical applicability is limited to the last months of pregnancy, when the fetus is already in a cephalic presentation. The clinical applicability of blood oxygen level dependent (BOLD) imaging, used for resting state functional MRI, was presented a few years ago, and presumably will contribute significantly to our understanding of the timing and mechanisms of early developmental abnormalities (18).

\section{Utility and limitations of fetal MRI}

Due to its proven utility, availability and low cost, ultrasound (US) remains the primary modality for fetal imaging. Fetal MRI has, however, several advantages over US. It has superior contrast and temporal resolution compared to US, it allows direct visualization of both hemispheres, while with US the anterior hemisphere is shadowed by reverberation artifacts from overlying structures. Decreased amniotic fluid, fetal position, and acoustic shadowing from ossifying calvaria also do not influence the quality of MR imaging, while polyhydramnios does (12). In cases when the mother is obese, image quality can be poor on US as well as on MRI, due to reduced SNR, however, MRI usually provides more image detail than US (13). Studies have shown that fetal MRI can detect up to $50 \%$ of sonographically occult pathologies (19, 20, 21). The limitations of MRI are fetal motion, the small size of the structure being imaged, the distance between the fetus and the receiver coil, any ferromagnetic material that cannot be removed, and also maternal claustrophobia (12).

\section{Development of the fetal brain}

Fetal brain development follows a predictable course, and familiarity with normal develop- 
mental anatomy from primary neurulation on is essential for proper interpretation of fetal MRI. There are several articles available that cover this topic $(22,23)$. MR imaging of the fetal brain is initially characterized by the presence of multiple layers that disappear as the brain matures and sulci form. A multi-layered pattern is visible on $\mathrm{T} 1$ and T2-weighted images from approximately 20 weeks until approximately 28 weeks (Fig. 2), characterized by bands of alternating $\mathrm{T} 1$ signal intensity between the germinal matrix and the developing cortex. The innermost, T1 hyperintense, T2 hypointense layer, is the ventricular zone or germinal matrix, the source of neuroectodermal elements that form the brain parenchyma. It gradually regresses towards the end of pregnancy, persisting in the caudothalamic groove through the early postnatal period. The ventricular zone is followed by the $\mathrm{T} 1$ hypointense periventricular zone, the T1 hyperintense subventricular and intermediate zones, the T1 hypointense subplate and T1 hyperintense cortex (2426). Sulcation of the fetal brain also follows a predictable course, and is one of the most accurate indicators of fetal gestational age (GA) (27) (Fig. 3). For proper assessment we

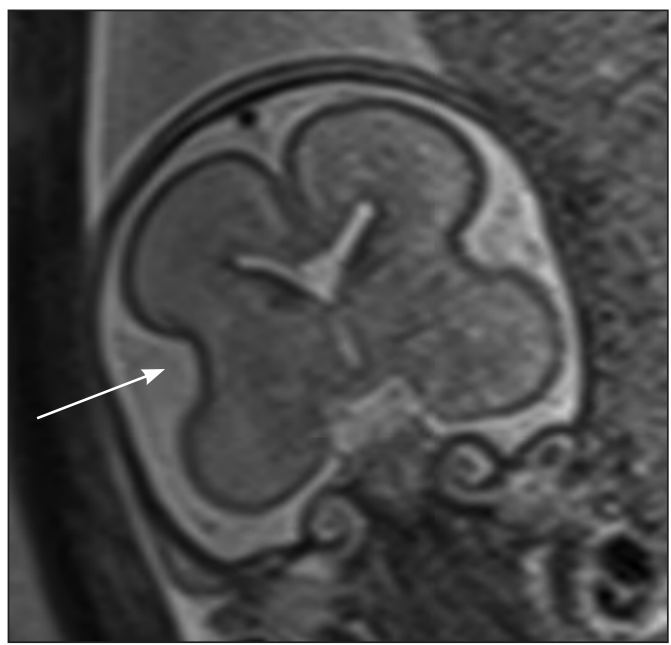

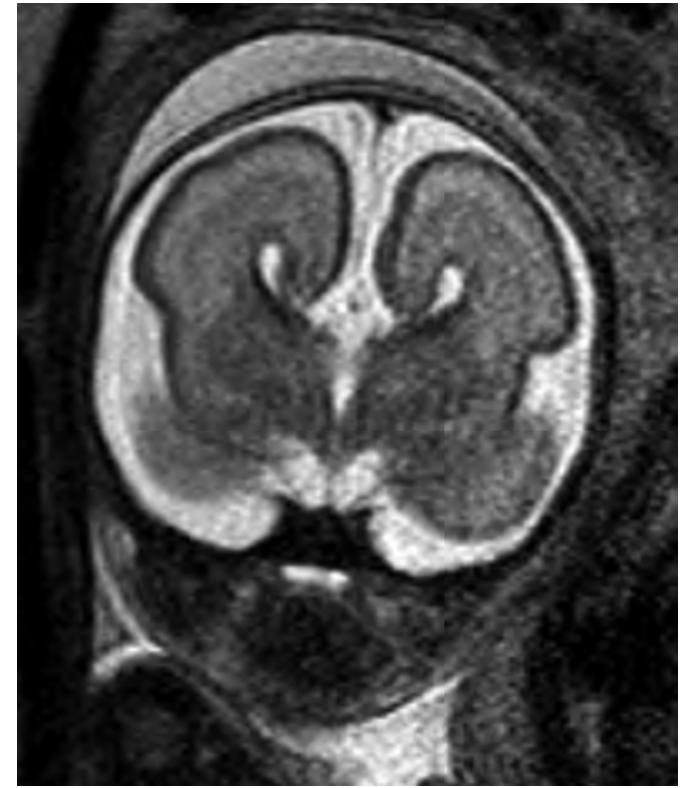

Fig. 2 A 26 week old fetus with isolated corpus callosum agenesis. Notice the typical multilayered pattern - alternating hypo- and hyperintense signals from the germinal matrix to the cortex (5 layers). T2 weighted sequence in the coronal plane.

have to know the gestational age of the imaged fetus and the timing of the appearing

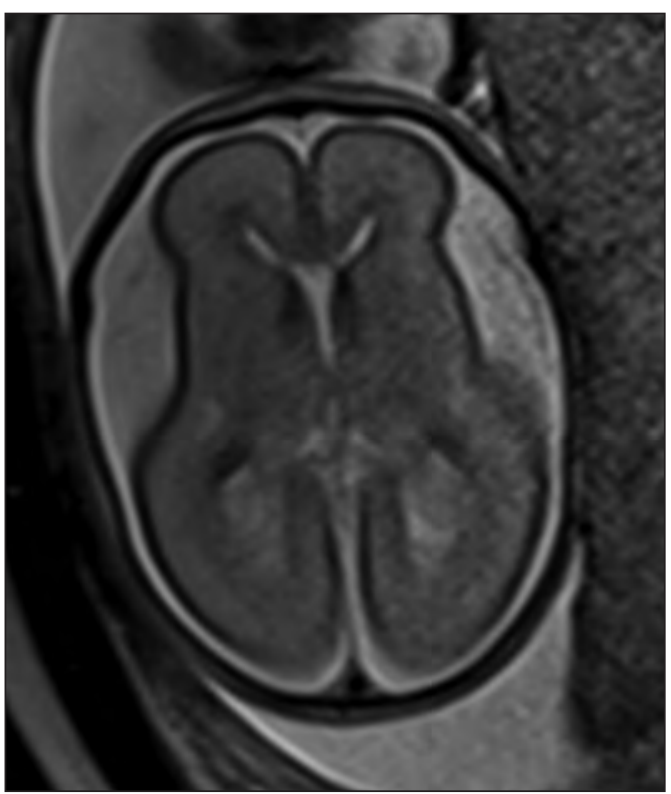

Fig. 3 A 22 week old fetus with no pathological findings intracranially. Notice the smooth surface of the brain with the exception of the infoldings of the Sylvian fissures (arrow). T2 weighted sequence in the coronal (left) and axial (right) planes. 
sulci. The first sulcus that appears as a small infolding before gestational week 18 , is a Sylvian fissure. At gestational week 34, all of the primary sulci and most of the secondary sulci are typically developed (27). Sulcation may be slightly delayed in twin pregnancies, and minor asymmetry between left and right hemisphere may be detected (28). Sulcation can also be delayed in fetuses with mild ventriculomegaly and other CNS pathologies (29). Ventricular size should be relatively constant between gestational weeks 15 and 35 (30). Atrial width, that is, the ventricular diameter measured through the posterior margin of the choroid plexus glomus at the level of the thalami in the axial plane, should be normally less than $10 \mathrm{~mm}$ (30). The ventricular walls should be smooth.

\section{Indications for fetal MRI}

The usual indications for fetal MRI include confirmation and characterization of CNS abnormalities detected on US, and detection of potential additional sonographically occult abnormalities. The most common indication for fetal MRI is ventriculomegaly diagnosed by US. Other common indications are: commissural abnormalities, infratentorial pathology, predominantly vermian agenesis or hypoplasia, cortical malformations, spinal malformations and complications of monochorionic twin pregnancies $(12,31)$. Assessment of other fetal structures, the umbilical cord and placenta, if visible, should also be undertaken.

\section{Ventriculomegaly}

Ventriculomegaly is the most common CNS pathology discovered by prenatal US examination. It is defined as an atrial diameter of $\geq 10 \mathrm{~mm}$. It is generally considered mild if the atrial diameter is between $10-14 \mathrm{~mm}$, moderate when the atrial diameter is $\geq 15$ $\mathrm{mm}$, with the thickness of the surrounding brain parenchyma $\geq 3 \mathrm{~mm}$ (Fig. 4), and severe when the atrial diameter is $\geq 15 \mathrm{~mm}$, with the thickness of the surrounding brain parenchyma $<3 \mathrm{~mm}$ (32). Another classification system is based on measurement of the atrial diameter alone. Mild ventriculomegaly is defined as an atrial diameter of 10-12 mm, moderate as an atrial diameter of $13-15 \mathrm{~mm}$, and severe as an atrial diameter of $>15 \mathrm{~mm}$ (33). The term "isolated ventriculomegaly" is used in cases when no other pathology is found at the time of diagnosis. Ventriculomegaly can be caused by a variety of disorders that may result in neurological, motor, and/ or cognitive impairment. They can roughly be divided into three categories (34):

1) An imbalance between the production and absorption of cerebrospinal fluid, of which the obstructive form (e.g. aqueductal stenosis) is most frequently observed;

2) Developmental abnormalities such as partial or complete agenesis of the corpus callosum, neuronal proliferation and migration disorders;

3) Destructive processes leading to loss of neuronal tissue (e.g. ischemia, bleeding, infection) (Fig. 5).

Unilateral ventriculomegaly is more often seen in destructive processes, whereas developmental anomalies are more characterized by bilateral broadening of the ventricles (35). The prognosis for a fetus with ventriculomegaly depends on the degree of ventricular dilatation and the presence of additional cerebral or extracerebral pathology $(33,34$, 36). In fetuses with isolated mild ventriculomegaly $(10-12 \mathrm{~mm})$ the possibility of a favorable postnatal outcome is up to $94 \%$ (37), while a fetus with additional anomalies and a larger atrial diameter has a much less favorable prognosis. Since the prognosis of fetal ventriculomegaly is related to the presence of additional abnormalities, the prenatal detection of such abnormalities is critical. With a MR study we can detect additional CNS anomalies in up to $70-85 \%$ of cases of fetal ventriculomegaly (19, 33-36). 

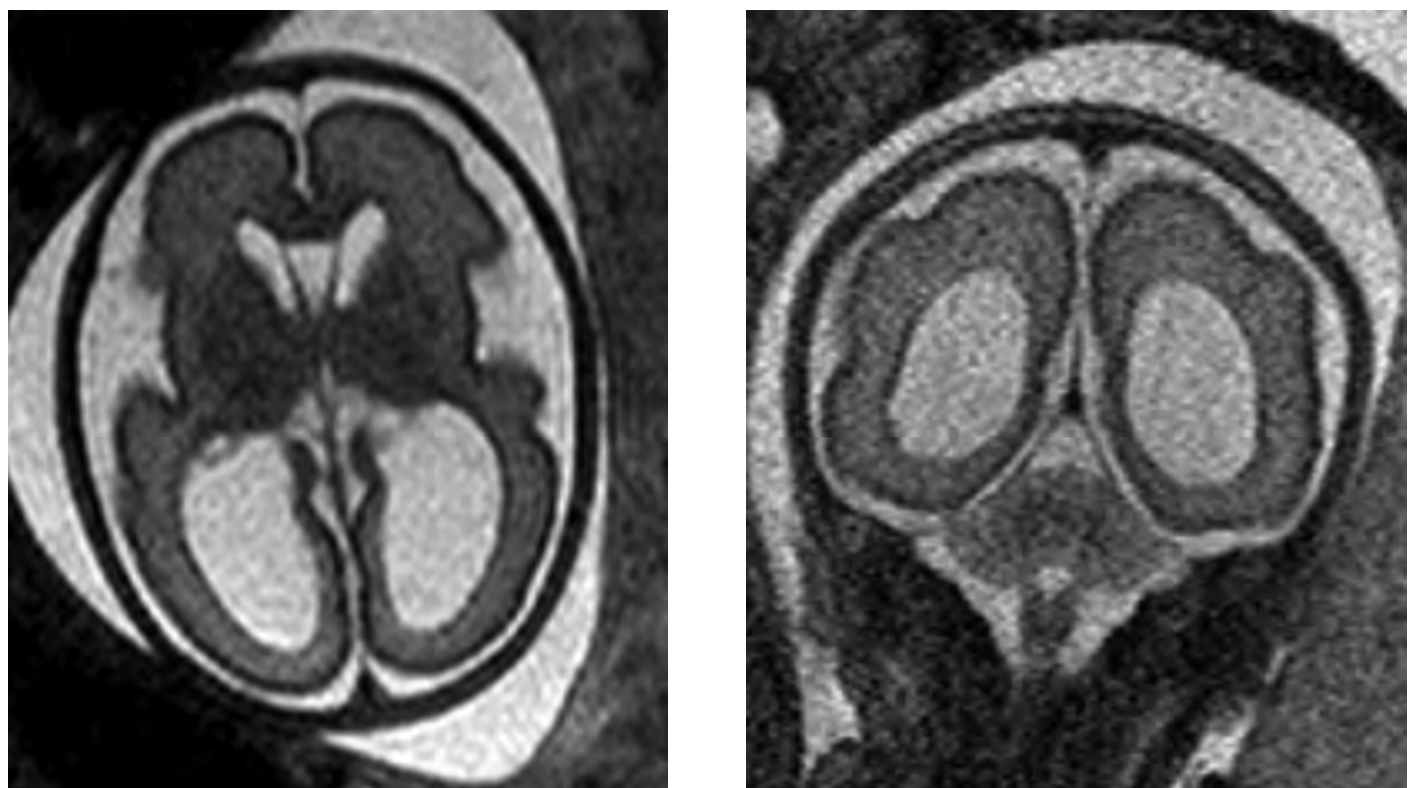

Fig. 4 A 28 week old fetus with isolated moderate bilateral ventriculomegaly and no other pathological findings. T2 weighted sequence in the axial (left) and coronal (right) planes.
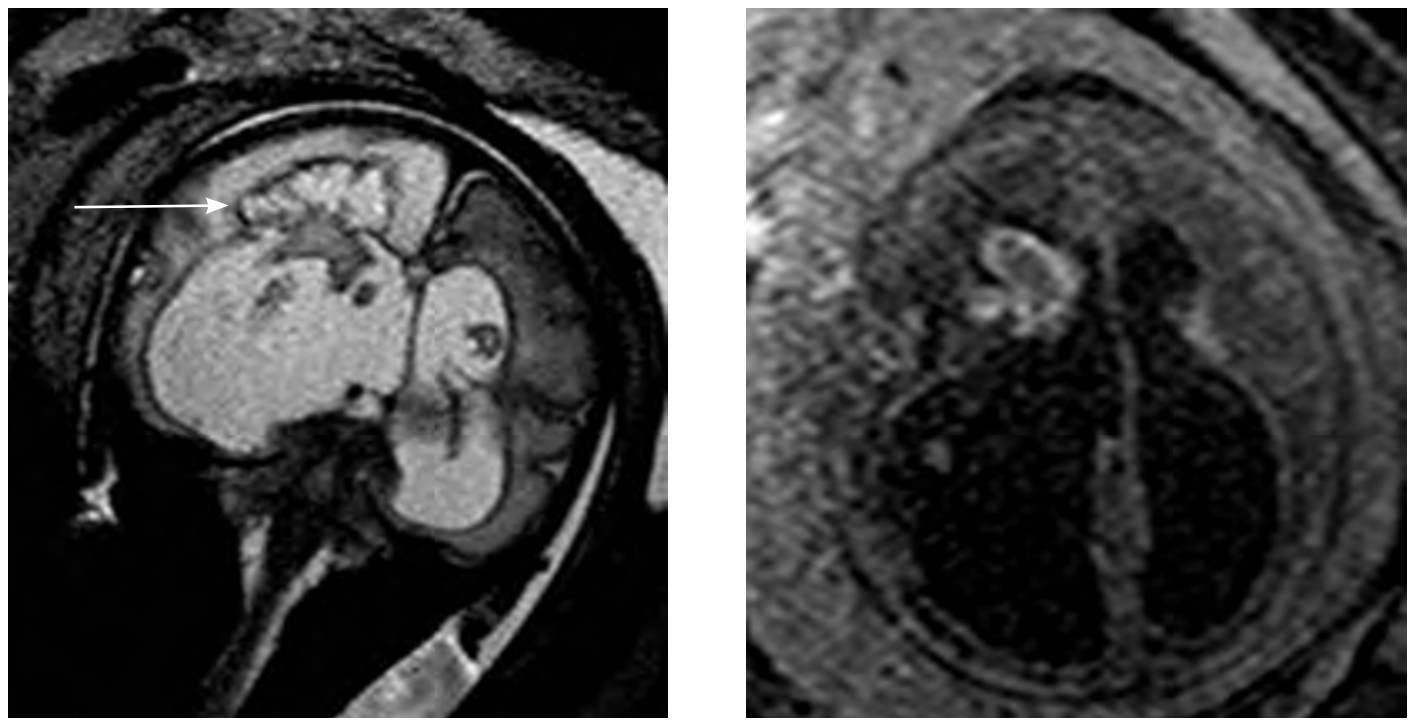

Fig. 5 A 33 week old fetus with severe bilateral asymmetric ventriculomegaly -hydrocephalus, periventricular white matter injury (arrow), parenchymal edema. A hyperintense blood clot on T1 weighted sequence (right). T2 weighted sequence in the coronal plane (left) and T1 weighted sequence in the axial plane (right).

\section{Commissural abnormalities}

Corpus callosum (CC) is the largest of the three major commissures that connect the left and right hemispheres. It develops between the $8^{\text {th }}$ and the $20^{\text {th }}$ gestational week.
CC agenesis is one of the most common congenital brain malformations. It may be complete or partial, and there may be dysgenesis, hypoplasia or destruction of the CC. Direct visualization of CC by US is difficult, while 
with fetal MRI it is possible to visualize CC directly in three planes. Abnormalities of the $\mathrm{CC}$ are rarely isolated (Fig. 6) and because the presence of associated intra or extracranial anomalies determines the future neurodevelopmental outcome $(38,39)$, it is important to detect them.
Fetal MRI can detect additional brain abnormalities in up to $63 \%$ of cases with callosal abnormalities that were detected on prenatal ultrasound. These include cortical malformations (Fig. 7), acquired pathologies and posterior fossa malformations $(39,40)$. It has been reported that in approximately
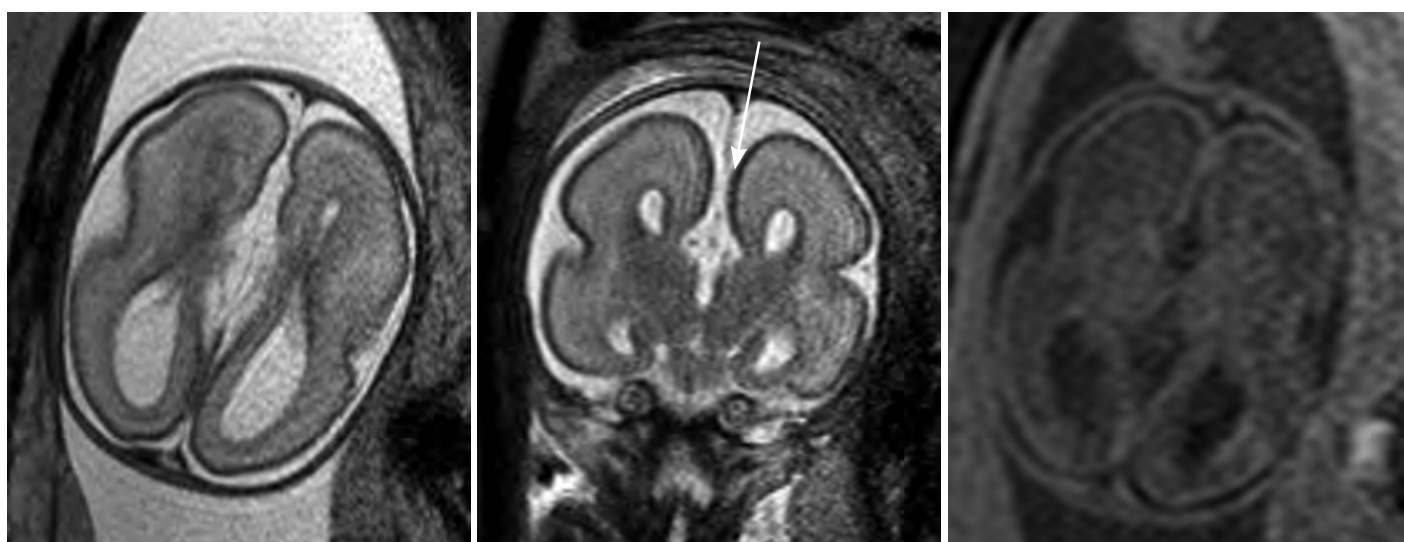

Fig. 6 A 26 week old fetus with isolated agenesis of the corpus callosum, teardrop configuration of the lateral ventricles on axial images (left), upward orientation of frontal horns and communication between the third ventricle and an interhemispheric fissure visible on coronal images (arrow). T2 weighted sequence in the axial plane (left), in the coronal plane (middle), T1 weighted sequence in the axial plane (right).
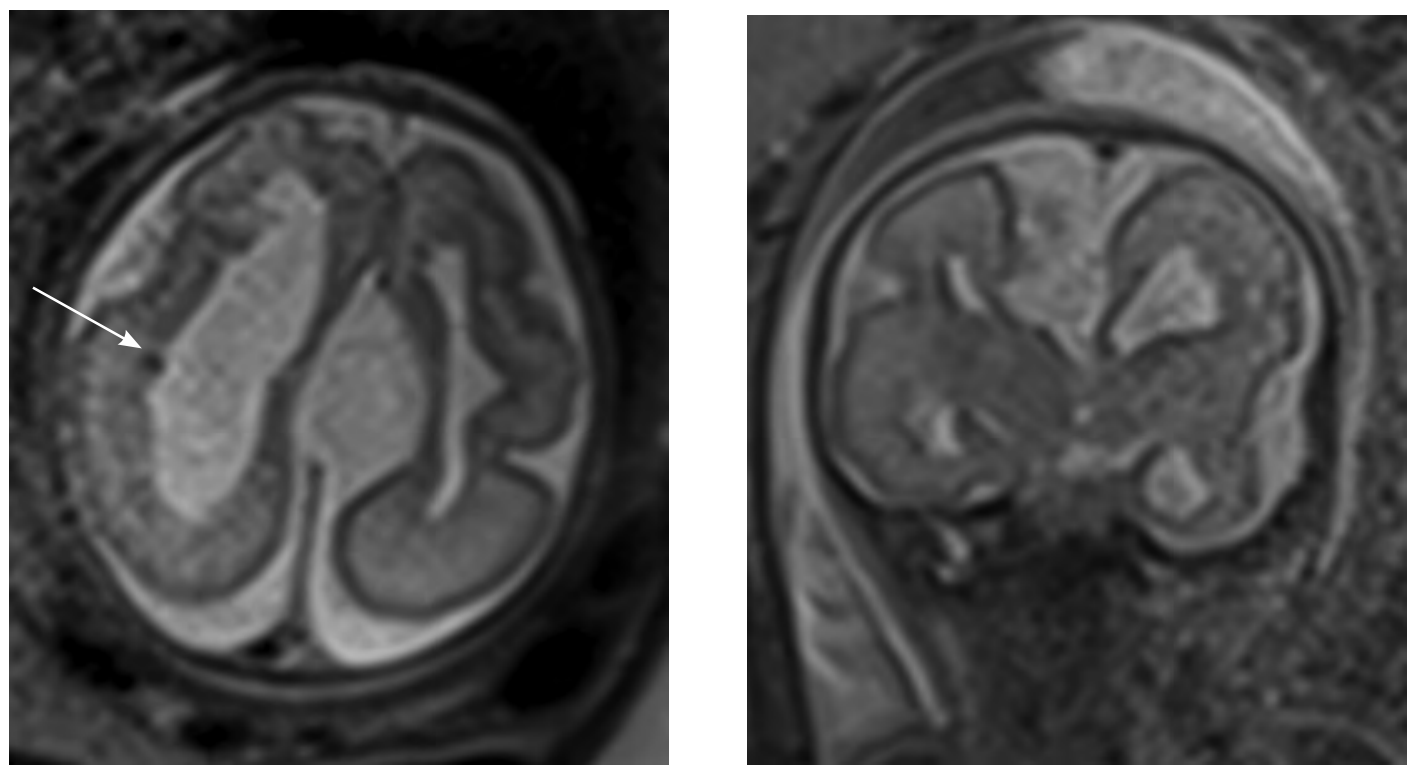

Fig. 7 A 27 week old fetus with agenesis of the corpus callosum and an inter-hemispheric cyst. Additional findings are: abnormal sulcation bilaterally (polymicrogyria), abnormal contours of the lateral ventricles, subependymal nodular heterotopia (arrow on the axial image). T2 weighted sequence in the axial plane (left) and the coronal plane (right). 
$20 \%$ of cases referred for callosal abnormalities detected by US, normal CC was found on fetal MRI (40). In difficult cases, DTI with MR tractography may depict commissures and misguided fiber tracts (41).

\section{Posterior fossa abnormalities}

Using fetal imaging and the pathogenesis of some abnormalities Garel et al. (42) divided the most common pathologies into 3 major groups:

1) Pathology with enlarged retrocerebellar fluid space and normal or abnormal biometry (Dandy-Walker malformations, Blake's pouch cyst, vermian agenesis);

2) Partially or globally decreased cerebellar biometry (cerebellar hypoplasia, agenesis, Rhombencephalosynapsis, ischemic and or hemorrhagic damage) (Fig. 8);

3) Abnormal partial or global cerebellar echogenicity (ischemic and/or hemorrhagic damage, cerebellar dysplasia, capillary telangiectasia).

US can detect major pathology of the infratentorial structures, while minor abnormalities are difficult to distinguish. Moreover, sonographic visualization of the posterior fossa is limited by ossification of the skull in the third trimester (31). Fetal MR imaging offers multidimensional analysis of cerebel- lum, vermis and brainstem (43). Appropriate timing for performing fetal MRI in suspected cerebellar and posterior fossa pathologies is also important (42).

\section{Malformations of cortical development}

Malformations of cortical development in a fetus often have similar appearance as cortical malformations in a child (Fig. 9). Due to its better contrast and temporal resolution MR imaging can detect cortical malformations that were not identified on US. The results of one study showed that MRI is superior in identifying schizencephaly, lissencephaly, polymicrogyria, and gray matter heterotopia in fetuses (44). It is important to be familiar with the normal appearance of fetal brain at different gestational ages to be able to identify these malformations.

\section{Spine anomalies}

Fetal MR imaging of spinal malformations typically serves as a secondary confirming or complementing imaging modality, after prenatal US has identified a spinal anomaly. It is a valuable additional tool in cases of maternal obesity, oligohydramnion, posterior position
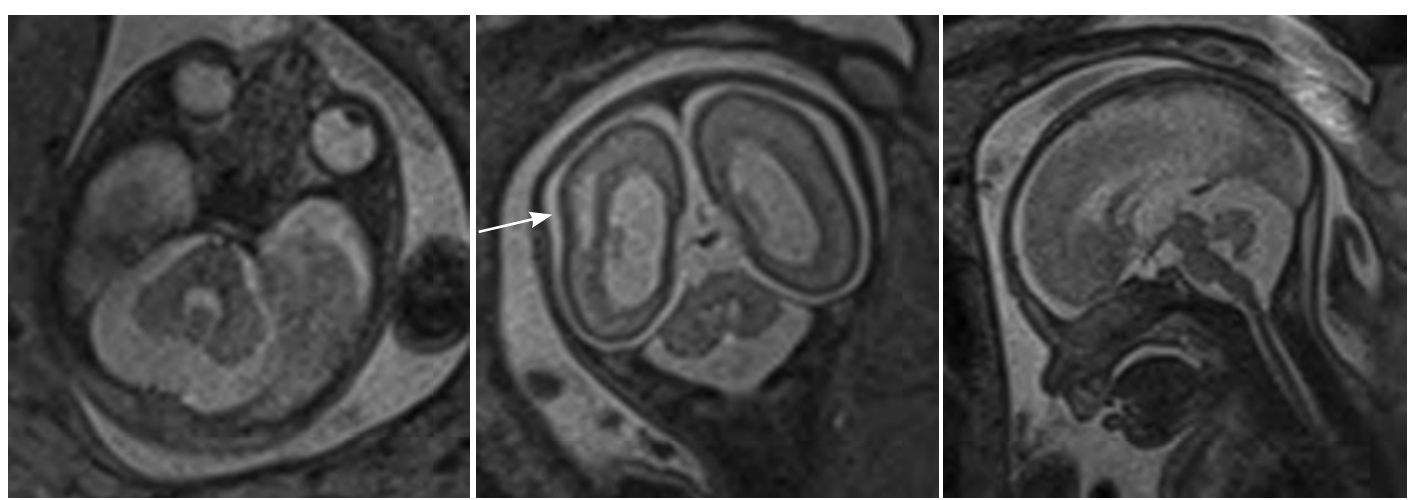

Fig. 8 A 26 week old fetus with hypoplastic cerebellar hemispheres and enlarged cisterna magna. Notice the enlarged lateral ventricles and the area of periventricular white matter injury in the right parietal area (arrow). T2 weighted sequence in the axial (left), coronal (middle) and sagittal (right) plane. 

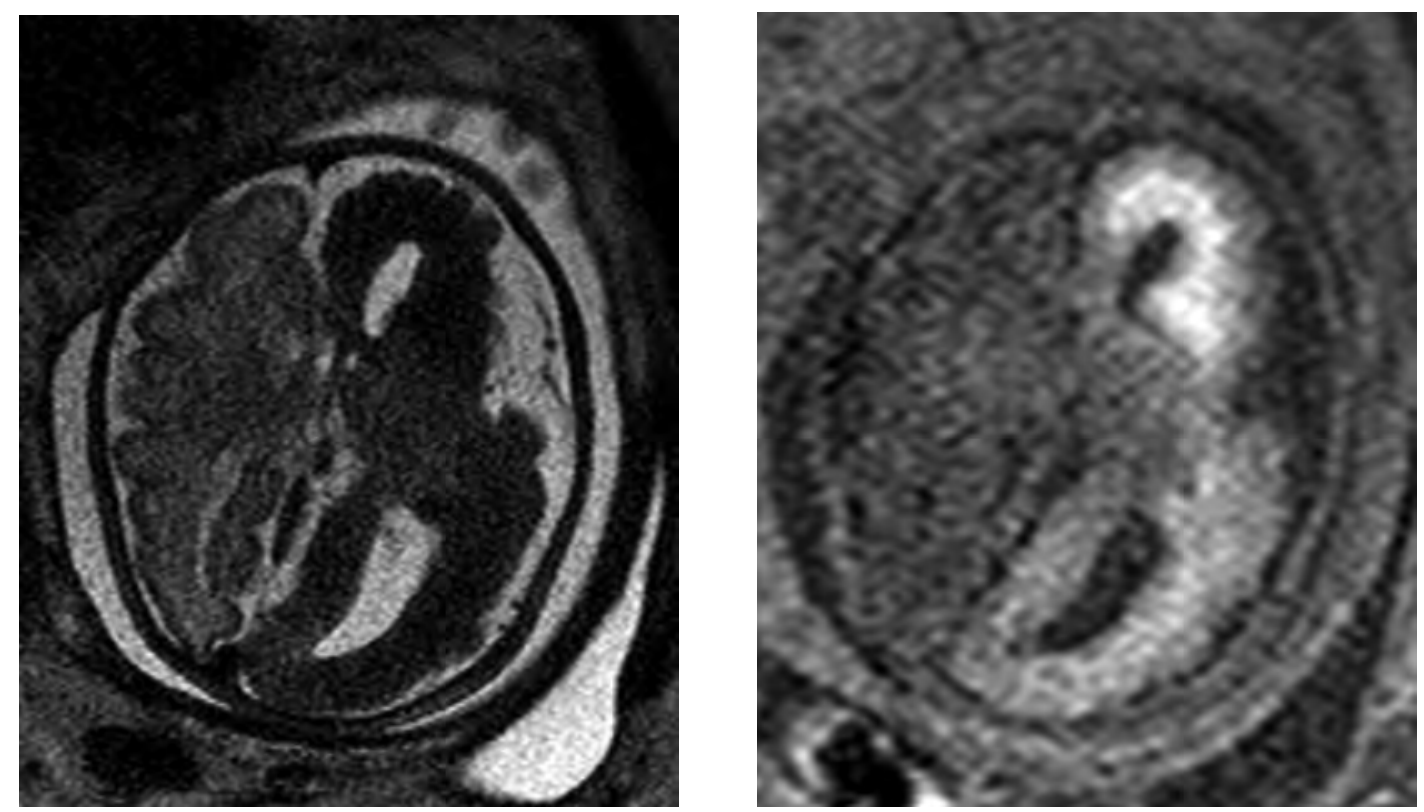

Fig. 9 A 32 week old fetus with an enlarged left hemisphere, with displacement across the midline, enlarged left lateral ventricle, abnormal sulcation and abnormal signal due to hypermyelinaton and calcifications of the left hemisphere - findings consistent with hemimegalencephaly confirmed on autopsy. T2 weighted sequence in the axial plane (left), T1 weighted sequence in the axial plane (right).

of the fetal spine with respect to the mother, in detecting additional intracranial malformations, as well as various uro-genital and gastro-intestinal malformations (45). Com-

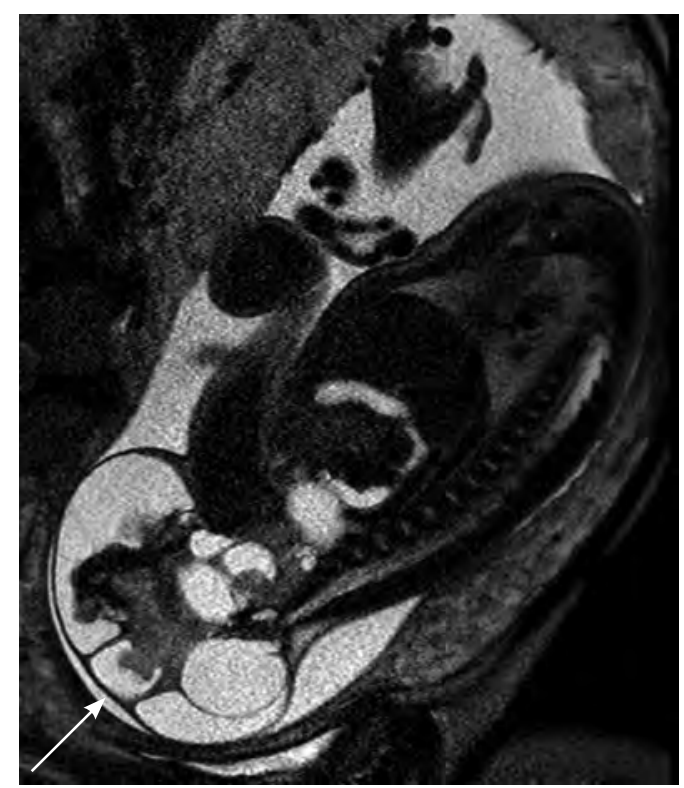

mon malformations of the spine include open or closed dysraphism, diastematomyelia, tethered cord, scoliosis and sacrococcygeal teratoma (Fig. 10, 11).

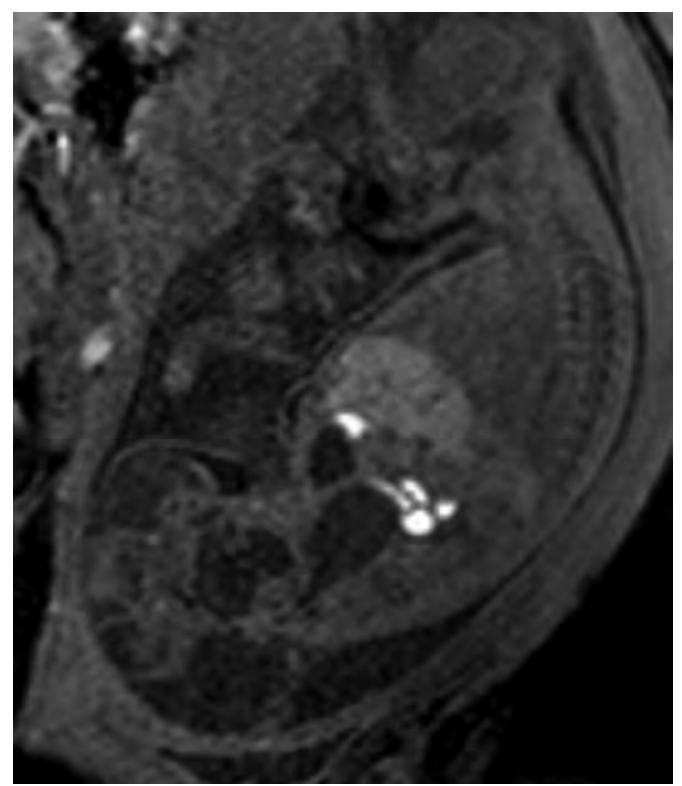

Fig. 10 A Large, partially cystic, partially solid extra and intrapelvic sacrococcygeal teratoma (arrow). T2 weighted sequence in the sagittal plane (left), T1 sequence in the sagittal plane (right). 

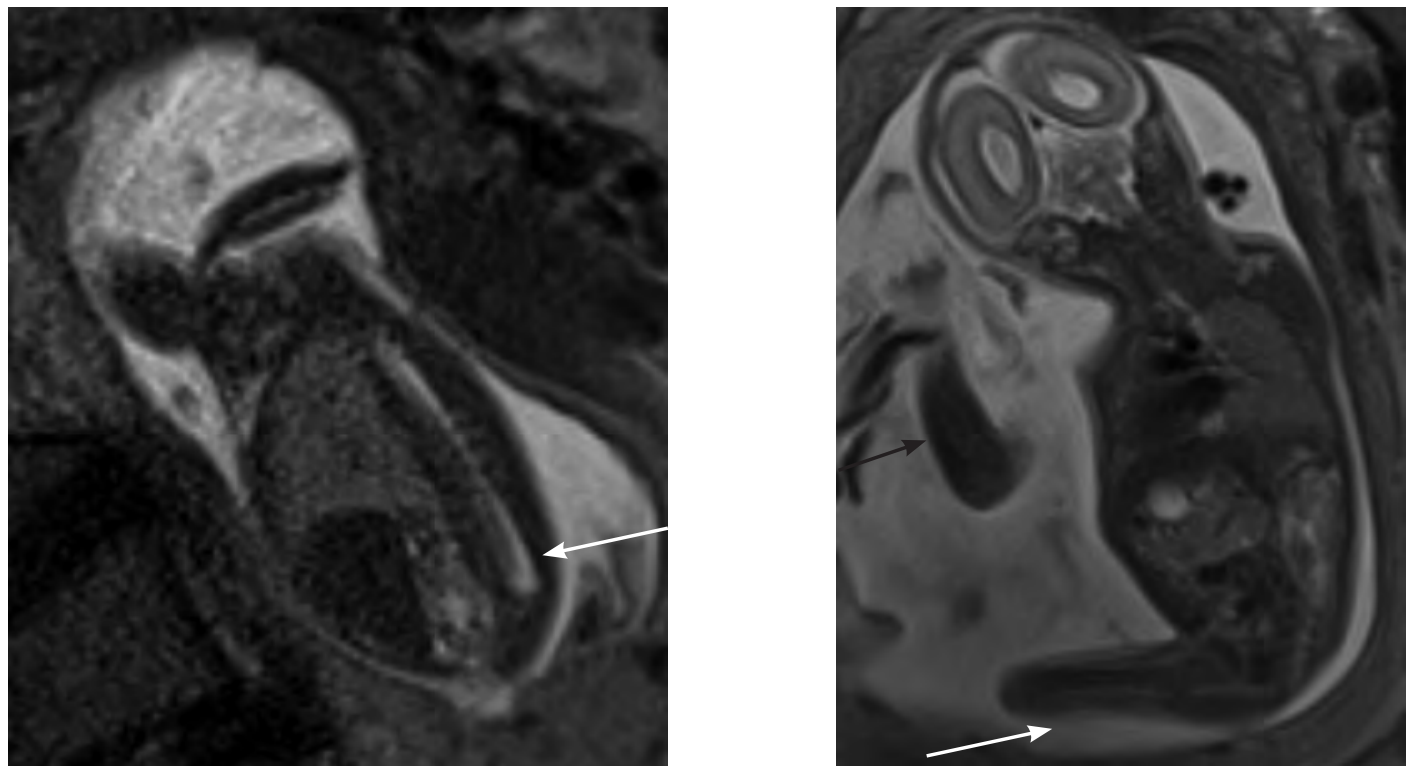

Fig. 11 Abrupt termination of the spinal cord above the expected level (left, arrow) and frog like or Buddha position of the legs with muscle atrophy. Compare the thickness and muscle signal of the leg (white arrow, right image) with the arm, visible on the same image (black arrow, right image) - caudal regression syndrome in a fetus of a diabetic mother. T2 weighted sequence in two planes.

\section{Complications of monochorionic twin pregnancies}

In complications of monochorionic twin pregnancy, such as co-twin demise, the surviving twin may suffer from acute cerebral hypoperfusion and/or thrombembolism (46).

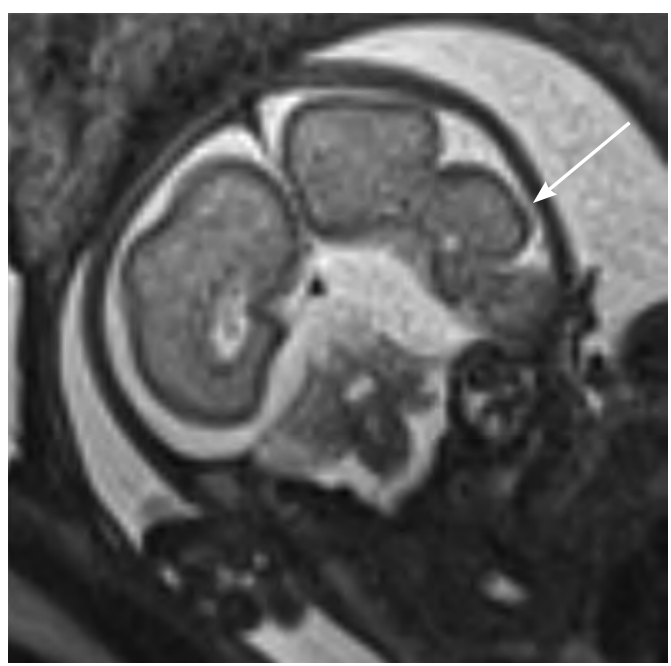

Sonographically, occult abnormalities can be detected in one-third of surviving twins, with abnormalities including polymicrogyria, encephalomalacia, germinolytic cysts, hemorrhage, ventriculomegaly, and delayed sulcation $(47,48)$. Another serious complication

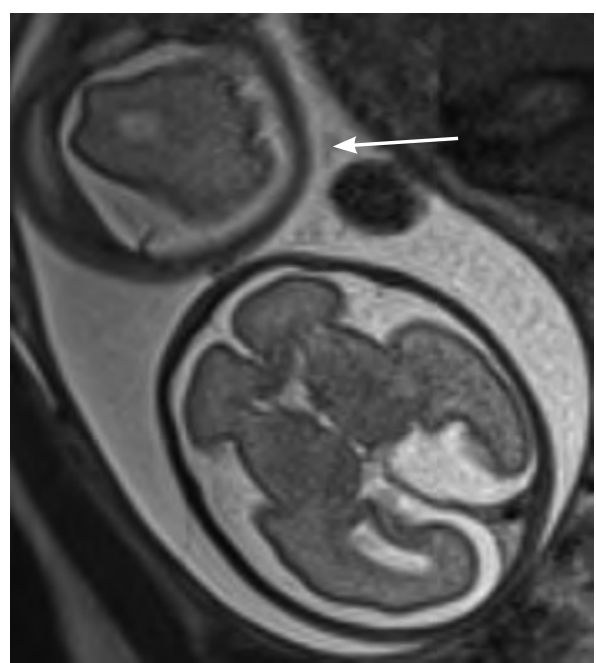

Fig. 12 A 28 week old monochorionic twin. There is cortical malformation of the left occipital lobe (arrow), and cystic dilatation at the medial aspect of occipital hemisphere. On the axial plane, part of the other twin's head is visible (small arrow). T2 weighted sequence in the axial plane (left) and in the coronal plane (right). 
of monochorionic twin pregnancies is twintwin transfusion syndrome. The morbidity rate in this syndrome is high, and both the donor and the recipient twin are at increased risk for cerebral ischemia and hemorrhage (47). Brain abnormalities detected by fetal MRI are similar to those seen in survivors of co-twin demise (Fig. 12). In both settings, MRI should be performed as early as possible to identify acute injury (48), and repeated a few weeks later to assess its chronic sequelae.

\section{Conclusion}

Fetal MRI is an increasingly important diagnostic modality in prenatal evaluation of fetal CNS anomalies. Over the last two decades we have seen significant progress in this field. With continued technical development and research advances, we may soon expect the improved quality of standard sequences, better clinical applications of advanced sequences, and a better understanding of fetal development and pathology. Familiarity with the applications and limitations of fetal MRI and multidisciplinary collaboration remain essential for a radiologist involved in prenatal imaging.

Conflict of interest: The author declares that she has no conflict of interest.

\section{References}

1. Smith FW, Adam AH, Philips WDP. NMR imaging in pregnancy. Lancet. 1983;1:61-2.

2. Angtuaco T, Shah H, Mattison D, Quirk J. MRI in high risk obstetric patients: a valuable complement to US. RadioGraphics. 1992;12:91-109.

3. Baker PN, Johnson IR, Gowland PA, Hykin J, Harvey PR, Freeman A, et al. Fetal weight estimation by echo-planar magnetic resonance imaging. Lancet. 1994;343(8898):644-5.

4. Kiefer B, Grassner J, Hausman R. Image acquisition in a second with half Fourier acquisition single-shot turbo spin-echo [abstract]. J Magn Reson Imaging. 1994;4 (Supp 1):86-7.
5. Levine D, Barnes PD, Sher S, Semelka RC, Li W, McArdle CR, et al. Fetal fast MR imaging: reproducibility, technical quality, and conspicuity of anatomy. Radiology. 1998;206:549-54.

6. Bouyssi-Kobar M, du Plessis AJ, Robertson RL, Limperopoulos C. Fetal magnetic resonance imaging: exposure times and functional outcomes at preschool age. Pediatr Radiol. 2015;45(12):182330.

7. Kanal E, Barkovich AJ, Bell C, Borgstede JP, Bradley WG Jr, Froelich JW et al. ACR guidance document on MR safe practices: 2013. Journal of Magnetic Resonance Imaging. 2013;37:501-30.

8. Patenaude Y, Pugash D, Lim K, Morin L. Diagnostic Imaging Committee: Lim K et al. The use of magnetic resonance imaging in the obstetric patient. J Obstet Gynaecol Can. 2014;36(4):349-63.

9. Heinrichs WL, Fong P, Flannery M, Heinrichs SC, Crooks LE, Spindle A, et al. Midgestational exposure of pregnant $\mathrm{BALB} / \mathrm{c}$ mice to magnetic resonance imaging. Magn Reson Imaging. 1988; 6:305-13.

10. Tyndall DA, Sulik KK. Effects of magnetic resonance imaging on eye development in the C57BL/6J mouse. Teratology. 1991;43:263-75.

11. Yip YP, Capriotti C, Talagala SL, Yip JW. Effects of MR exposure at $1.5 \mathrm{~T}$ on early embryonic development of the chick. J Magn Reson Imaging. 1994;4:742-8.

12. Glenn OA, Barkovich AJ. Magnetic resonance imaging of the fetal brain and spine; an increasingly important tool in prenatal diagnosis, part 1 . Review Article. AJNR Am J Neuroradiol. 2006;27;160411.

13. Prayer D. Fetal MRI. 1st ed.Springer-Verlag: Berlin Heidelberg 2011.

14. Glover P, Hykin J, Gowland P, Wright J, Johnson I, Mansfield P. An assessment of the intrauterine sound intensity level during obstetric echoplanar magnetic resonance imaging. Br J Radiol. 1995;68(814):1090-4.

15. Garel C. Fetal MRI: what is the future? Ultrasound Obstet Gynecol. 2008;31:123-8.

16. Kasprian G, Brugger PC, Weber M, Krssak M, Krampl E, Herold C, et al. In utero tractography of fetal white matter development. Neuroimage. 2008;43(2):213-24

17. Pugash D, Krssak M, Kulemann V, Prayer D. Magnetic resonance spectroscopy of the fetal brain. Prenat Diagn. 2009;29(4):434-41. 
18. Schopf V, Kasprian G, Schwindt J, Kollndorfer K, Prayer D. Visualisation of resting-state networks in utero. Ultrasound Obstet Gynecol. 2012;39(4)487-8.

19. Levine D, Barnes PD, Madsen JR, et al. Central nervous system abnormalities assessed with prenatal magnetic resonance imaging. Obstet Gynecol. 1999;94:1011-19.

20. Simon EM, Goldstein RB, Coakley FV, Filly RA, Broderick KC, Musci TJ, et al. Fast MR imaging of fetal CNS anomalies in utero. AJNR Am JNeuroradiol. 2000;21(9):1688-98.

21. Gonçalves LF, Lee W, Mody S, Shetty A, SangiHaghpeykar H, Romero R. Diagnostic Accuracy of Ultrasonography and Magnetic Resonance Imaging for the Detection of Fetal Anomalies - A Blinded Case-control Study. Ultrasound Obstet Gynecol. 2015;Oct 6. [Epub ahead of print].

22. Griffiths P. Atlas of fetal and postnatal brain MR. 1st ed. Philadelphia: Mosby/Elsevier; 2010.

23. Garel C. MRI of the fetal brain; Normal Development and Cerebral Pathologies. 1st ed. SpringerVerlag: Berlin Heidelberg; 2004.

24. Girard N, Raybaud C. In vivo MRI of fetal brain cellular migration. J Comput Assist Tomogr. 1992;16:265-67.

25. Girard N, Raybaud C, Poncet M. In vivo MR study of brain maturation in normal fetuses. AJNR Am J Neuroradiol. 1995;16:407-13.

26. Kostović I, Judas M, Rados M, Hrabac P. Laminar organization of the human fetal cerebrum revealed by histochemical markers and magnetic resonance imaging. Cereb Cortex. 2002;12(5):536-44.

27. Garel C, Chantrel E, Brisse H, Elmaleh M, Luton D, Oury JF, et al. Fetal cerebral cortex: normal gestational landmarks identified using prenatal MR imaging. AJNR Am J Neuroradiol. 2001;22;18489.

28. Chi J, Dooling E, Gilles F. Gyral development of the human brain. Ann Neurol. 1977;86-93.

29. Levine D, Barnes PD. Cortical maturation in normal and abnormal fetuses as assessed with prenatal MR imaging. Radiology. 1999;210:751-8.

30. Glenn OA, Barkovich AJ. Magnetic resonance imaging of the fetal brain and spine; an increasingly important tool in prenatal diagnosis, part 2. Review Article. AJNR Am J Neuroradiol. 2006;27;180714.
31. Levine D, Barnes PD, Robertson RR, et al. Fast MR imaging of fetal central nervous system abnormalities. Radiology. 2003;229:51-61.

32. Cardosa JD, Goldstein RB, Filly RA. Exclusion of fetal ventriculomegaly with a single measurement: the width of the lateral ventricular atrium Radiology. 1988;169(3):711-4.

33. Griffiths PD, Reeves MJ, Morris JE, Mason G, Russell SA, Paley MN, Whitby EH. A prospective study of fetuses with isolated ventriculomegaly investigated by antenatal sonography and in utero MR imaging. AJNR Am J Neuroradiol. 2010;31(1):106-11.

34. Cardoen L, De Catte L, Demaerel P, Devlieger R, Lewi L, Deprest J, et al. Claus F. The role of magnetic resonance imaging in the diagnostic work-up of fetal ventriculomegaly. Facts Views Vis Obgyn. 2011;3(3):159-63.

35. Girard N, Ozanne A, Chaumoitre K, Sigaudy S, Gire C, Dubuc M, et al.MRI and in utero ventriculomegaly. J Radiol. 2003;84(12 Pt 1):1933-44.

36. Pier DB, Levine D, Kataoka ML, Estroff JA, Werdich XQ, Ware J, et al. Magnetic resonance volumetric assessments of brains in fetuses with ventriculomegaly correlated to outcomes. J Ultrasound Med. 2011;30(5):595-603.

37. Falip C, Blanc N, Maes E, Zaccaria I, Oury JF, Sebag G, et al. Postnatal clinical and imaging follow-up of infants with prenatal isolated mild ventriculomegaly: a series of 101 cases. Pediatr Radiol. 2007;37(10):981-9.

38. Gupta JK, Lilford RJ. Assessment and management of fetal agenesis of the corpus callosum. Prenat Diagn. 1995;15:301-12.

39. Tang PH, Bartha ME, Norton ME, Barkovich AJ, Sherr EH, Glenn OA. Agenesis of the corpus callosum: an MR imaging analysis of associated abnormalities in the fetus. AJNR Am J Neuroradiol. 2009;30:257-63.

40. Glenn OA, Goldstein RB, Li KC, Young SJ, Norton ME, Busse RF, Goldberg JD, Barkovich AJ. Fetal magnetic resonance imaging in the evaluation of fetuses referred for sonographically suspected abnormalities of the corpus callosum. J Ultrasound Med. 2005;24(6):791-804.

41. Kasprian G, Brugger PC, Schopf V, Mitter C, Weber M, Hainfellner JA, et al. Assessing prenatal white matter connectivity in commissural agenesis. Brain. 2013;136(Pt 1):168-79. 
42. Garel C, Fallet-Bianco C, Guibaud L. The fetal cerebellum: development and common malformations. J Child Neurol. 2011;26(12):1483-92.

43. Vatansever D, Kyriakopoulou V, Allsop JM, Fox M, Chew A, Hajnal JV, et al. Multidimensional analysis of fetal posterior fossa in health and disease. Cerebellum. 2013;12(5):632-44.

44. Sonigo PC, Rypens FF, Carteret M, Delezoide AL, Brunelle FO. MR imaging of fetal cerebral anomalies. Pediatr Radiol. 1998;28:212-22.

45. Huisman TAGM, Graham EM, Tekes A, Poretti A. MRI of fetal spinal malformations. J Ped Neuroradiol. 2012:211-3.
46. van Heteren CF, Nijhuis JG, Semmekrot BA, Mulders LG, van den Berg PP. Risk for surviving twin after fetal death of co-twin in twin-twin transfusion syndrome. Obstet Gynecol. 1998;92(2):215-9.

47. Jelin AC, Norton ME, Bartha AI, Fick AL, Glenn OA. Intracranial magnetic resonance imaging findings in the surviving fetus after spontaneous monochorionic cotwin demise. Am J Obstet Gynecol. 2008;199(4):398.e1-5.

48. Hoffmann C, Weisz B, Yinon Y, Hogen L, Gindes L, Shrim A, Sivan E, Schiff E, Lipitz S. Diffusion MRI findings in monochorionic twin pregnancies after intrauterine fetal death. AJNR Am J Neuroradiol. 2013;34(1):212-6. 